erfahrenter und tüttiger Säger, fo bab bas wertden wohl allen, Dte mit

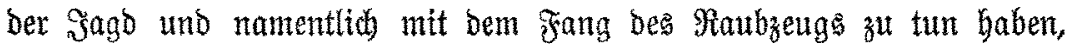
empfohlen werben fant.

Einige Kleine Beanitanoungett mögen ung aber boch geitattet fetn.

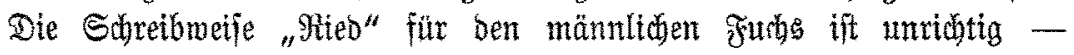

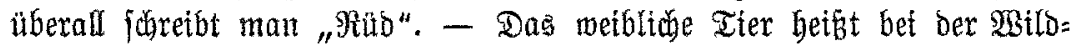
lake nicht Fähe, fonbern Rabe, währeno bas männlidje Rater ober

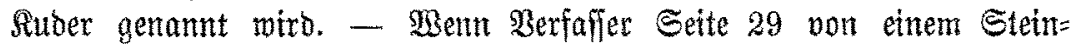
marberpaar mit 4 Sungen fpricht, fo mobte id bon bemerken, ba

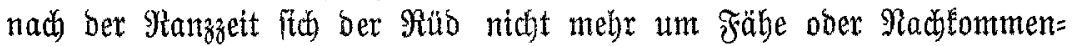
fofaft fümmert! Sette 36 wiro als äuberft intereffantes Faftum hervor:

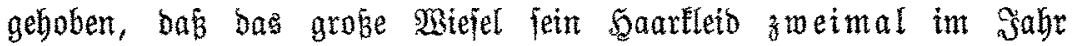
wedfele - bies ift boch bet allen unjern jagboaren Iieren ber Fall! Die Britezeit bes Buffiards wiro mit 5 möhentlicher Dauer angegeben

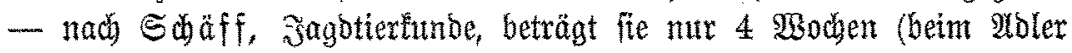
aber 5).

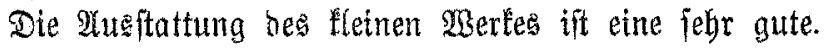

Dr. Fürit.

\title{
IV. Matizen.
}

\section{Staatsrat Dr. von Dorrer $\uparrow$.}

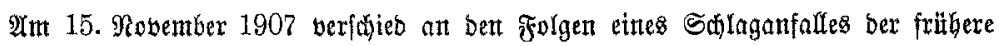

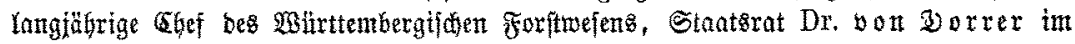

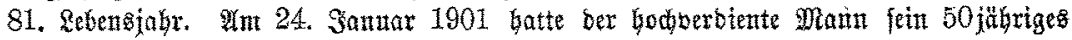

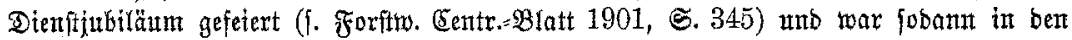

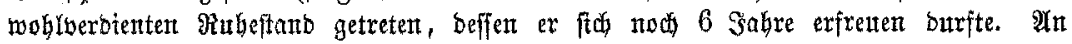

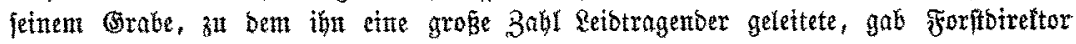

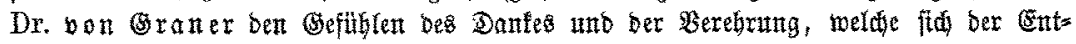
fallafene in weiten Exeifen und wor aflem bet jeitten Fodigenoffen ermorben hatte, marmen

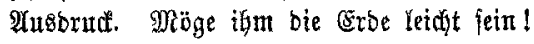

$\mathrm{F}$.

\section{Der franffurter ZZtbenbaum.}

Mitgeteilt yom (seb. Forftrat Reif in Sffenbad a. Main.

Das Sntereffe, welobe man gegenmärtig fogenannten Raturbentmälern entgegen= zubringen pflegt, worunter auch Bäume zu berftebent fint, beren Erbaltung ans ge=

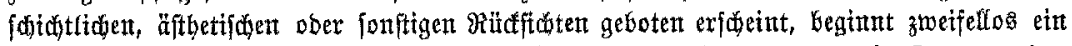

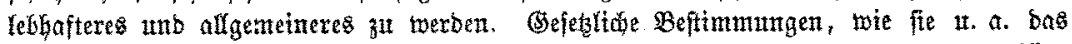

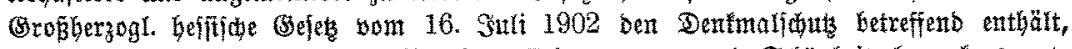

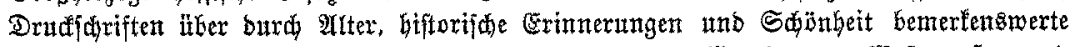

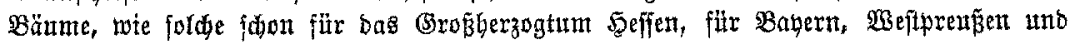




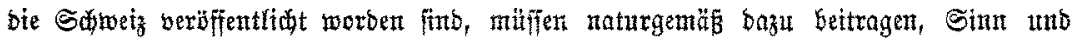

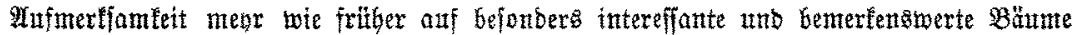

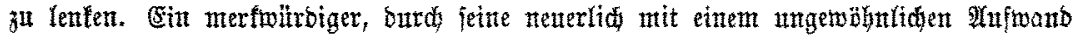

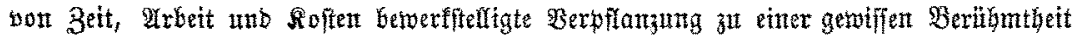
gefangter Bantm hatte feither fetnen Stanbont in Detn zur Dr. Senlenbergidiett

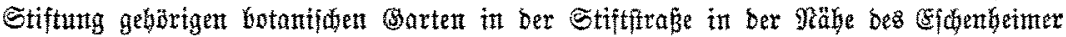
Tume zu Franffurt a. Main. (Es hanbelt fir un eine Eibe (Taxus baceata), bte

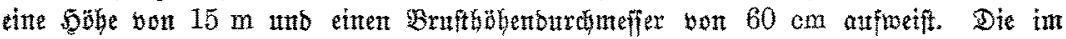

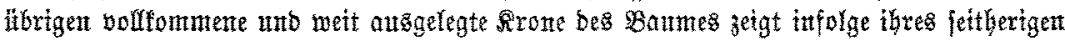

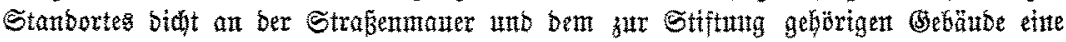

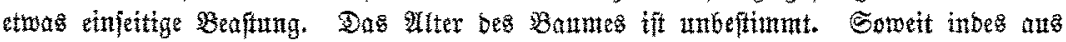

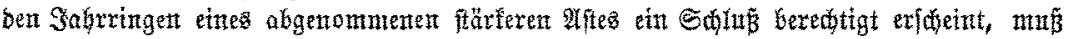

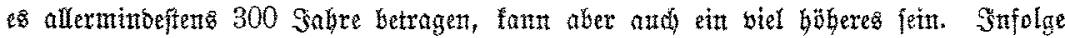

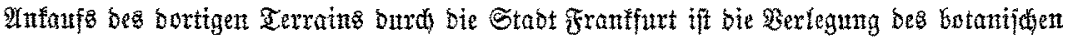

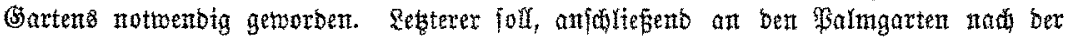

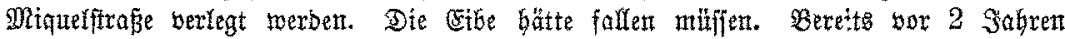

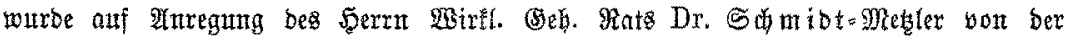

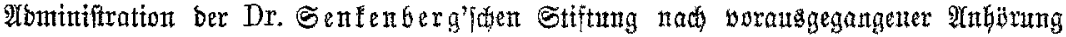

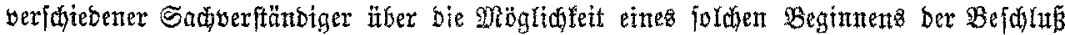

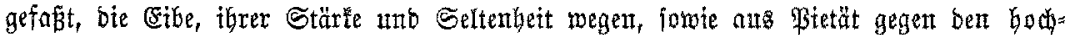

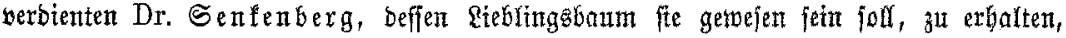

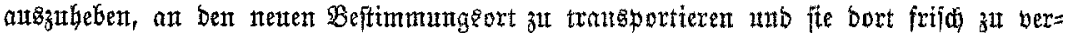
pffanzer. Mianderlei Sorarbeitent waren zut biejen Unternebnen erforberfid. Safon

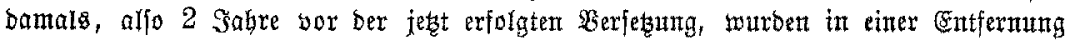

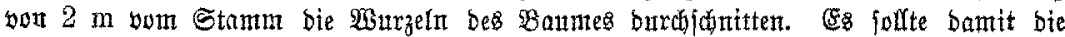

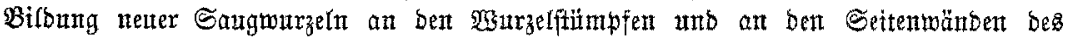

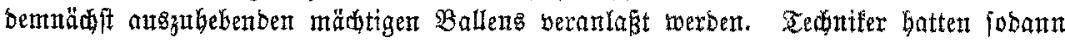

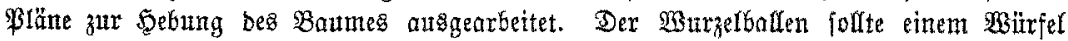
entfpreden, Deffen Seitenlänge $4 \mathrm{~m}$ unb beffen Tiefe $2 \mathrm{~m}$ betritg. Das (Semidit bon Baum und Balfen war anf ca. 900 3entnex berechut. Sn Englant betreibt eitte Firma

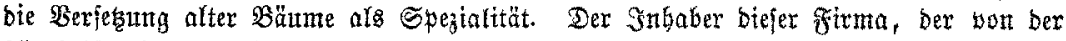
Ifominiftration bes Senfenberg'fiden Stifts erfudit toorben war, bie serbältniffe an

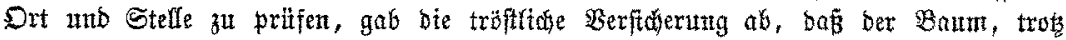

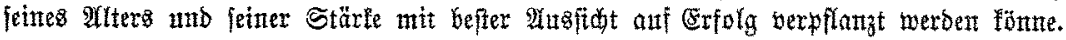

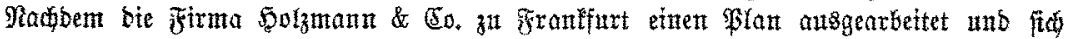

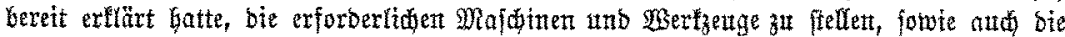
Sebuttg unb ben Trangport Des Banmes zit itbernebmen, wurbe numebr rilhrig an bie

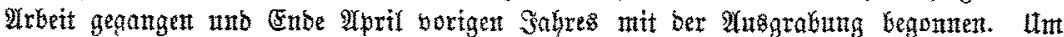

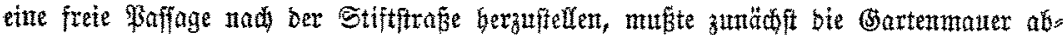
gebrodent werben. Sin weiten Infrete muste ber Boben bis zut einer Tiefe bon $3,10 \mathrm{~m}$

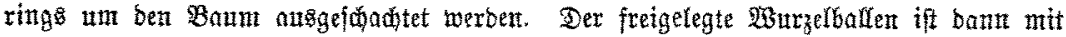

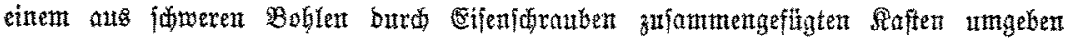

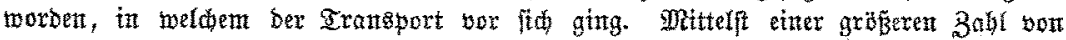

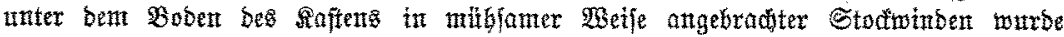

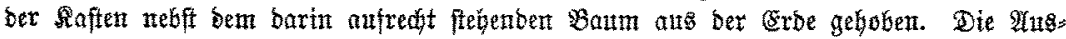
grabunggarbeiten batten faft volle 3 Boden gemähet. Mm 22. Mat beganth bex

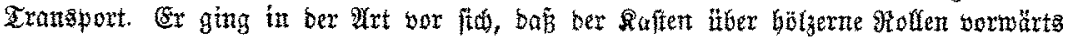

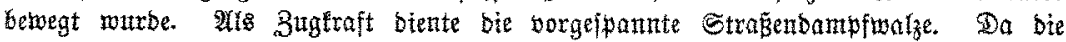


अollen auf bem mit Boblen belegten weg inmer wieber bor binten nadi bon thtter

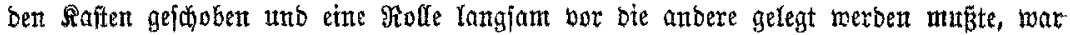

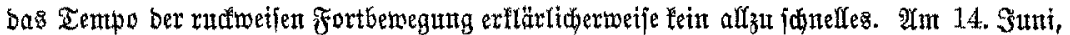

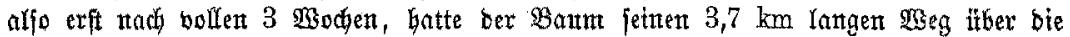

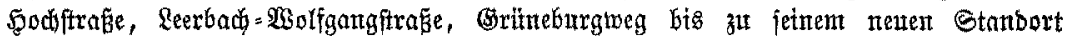

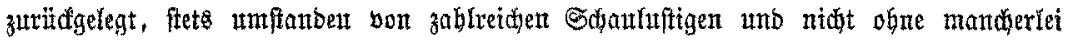

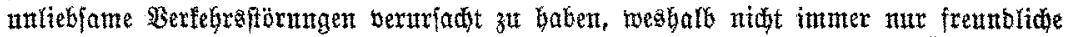

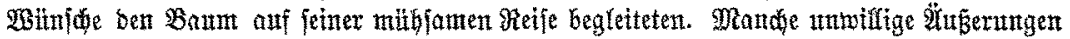
waren gu bertukmen, wenn Ungebulbige, wentger baumfrembliche Baffantet ibrem

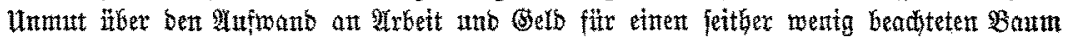

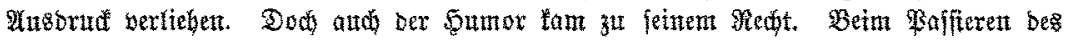

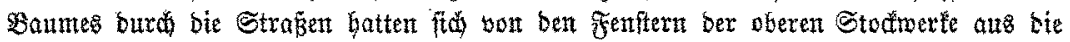

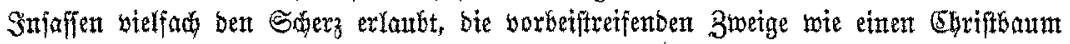

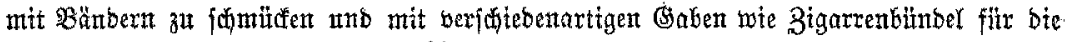

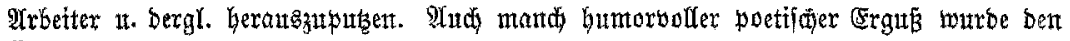

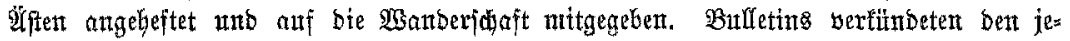

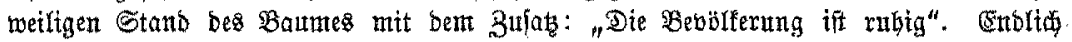

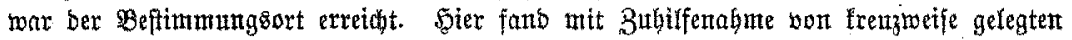

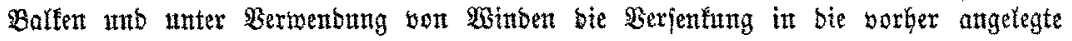

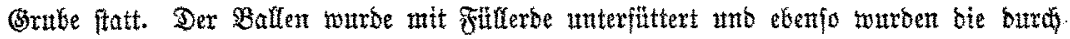

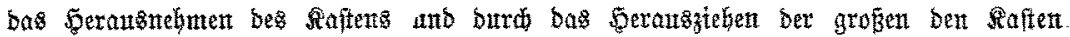

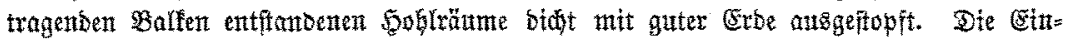

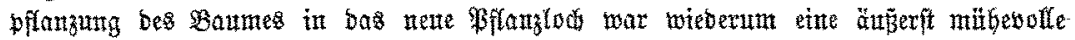

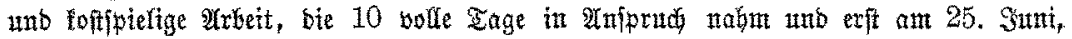

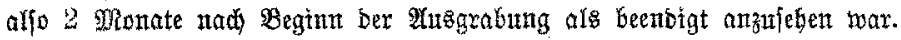

Eo war benn bas grop̉e werf sollbradit. Der Baum fteḅt jeit biefer Beit feft

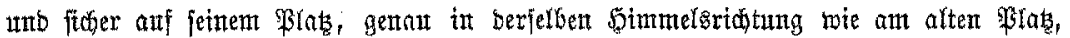

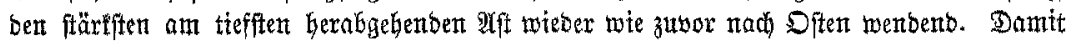
ber Stummino fein lunbeil anridten fath, Gat man ben Stamm foriorgitu mit

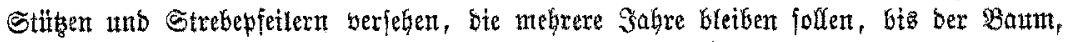

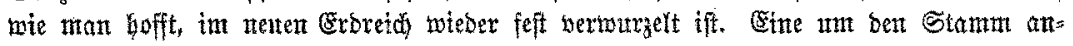

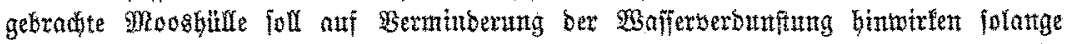

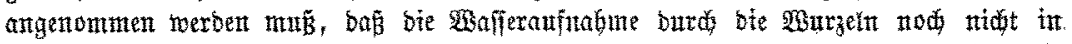

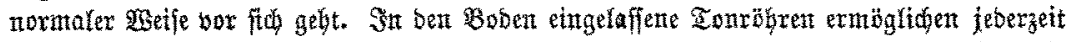

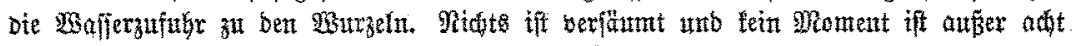

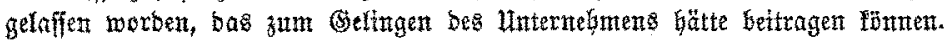

W3irb Der Banth bie Berpflanzung vortragen ind wirb ex weiter wadjen? Die

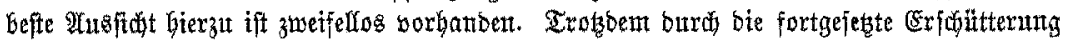

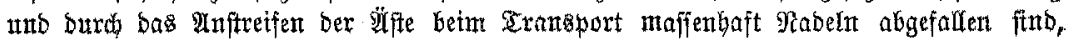

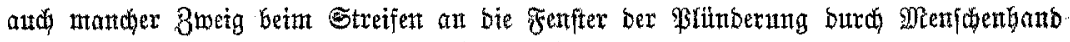

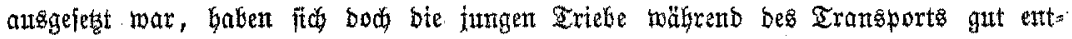

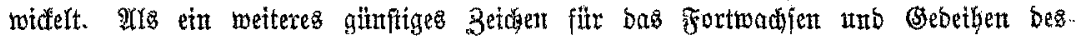

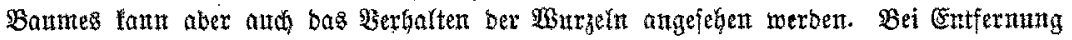

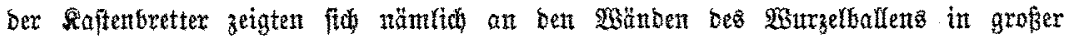

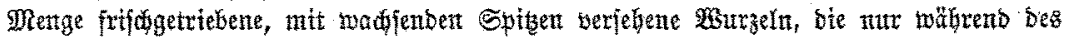

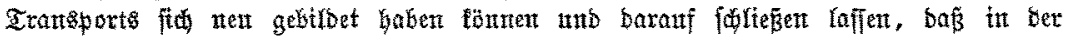

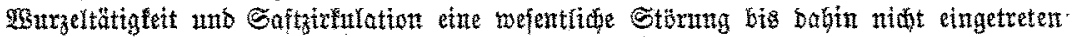

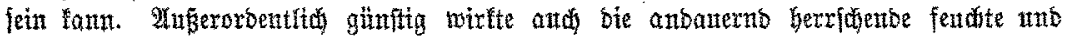




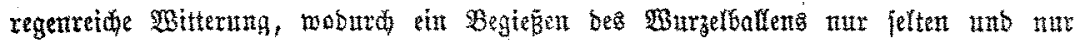
bann notwenbig war, wenn fith an Eroproben, bie man aus gubtern in ben Seitent wänben Geransnabm, ein gewifjer Sroctentheitsugrab bemertbar madte.

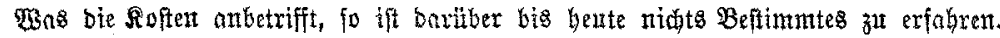

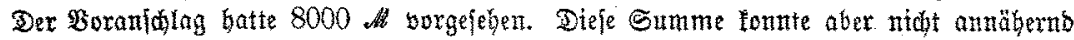

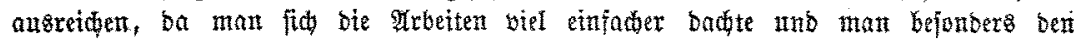
Transport in 3 ragen anflatt wie nadfer in 3 Boben zu bewertifelligen boffte. Es

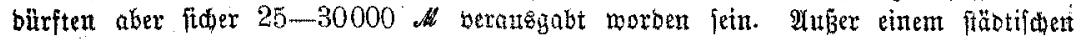
3ujwuß son 2000 . Alt ift bie ganze Summe burd pribate, freimillige Beiträge auf= gebradt worben.

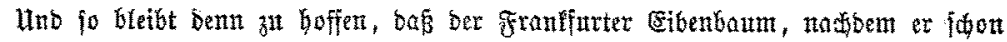

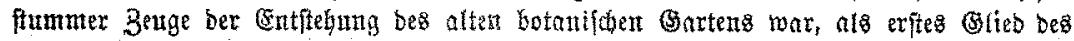

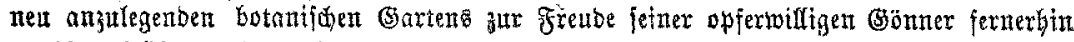
toadjent, blïben and gebeiben möge.

\title{
Driginalernteberth̨t über Kadel= und Eaubholjjamen 1907/08.
}

\author{
Bon Con rab wpel in Darmftast.
}

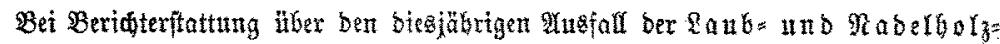

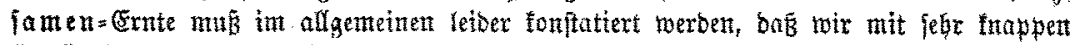
Erträgniffen zu redinet baven.

Stieleidife bat in unferem etrgen $\mathfrak{B a t e r f a m b e}$ feine Maft ergeben, nur bie

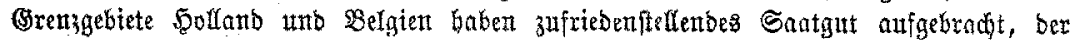
Serbfbebmi tonnte bamit gebedt werben, einige $\$$ soften wurben and wieber in gutes Binterlager genwamen.

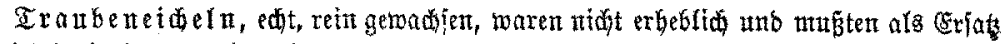

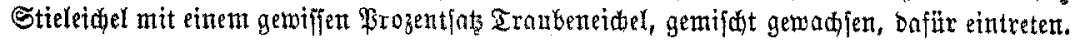

Moteicheln haben eine febr fleine Ernte gezeitigt, bie Sualität ift zufriebenftellent,

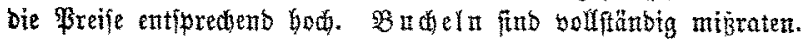

Die ïbrigen Raubbolzjamen batten teils unter bem folten Fritbjaht fu leiben;

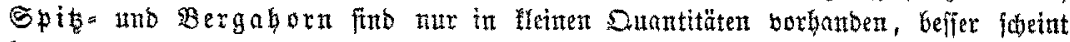

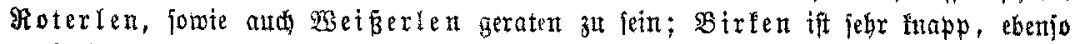

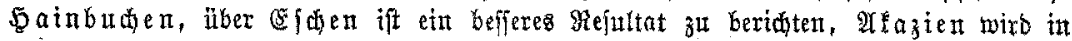

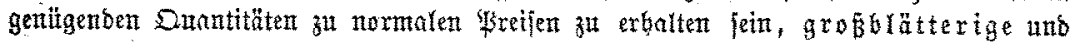

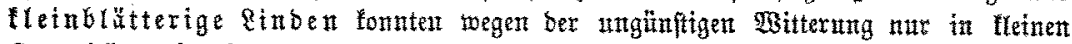
Snantitäten eingebradit werben.

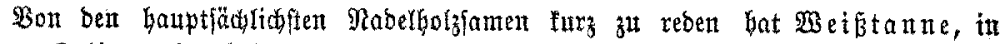

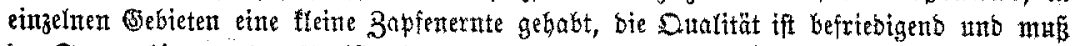

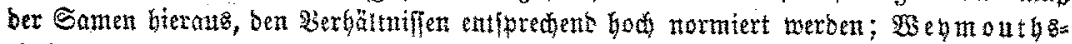

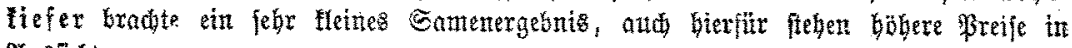
Irtoftert.

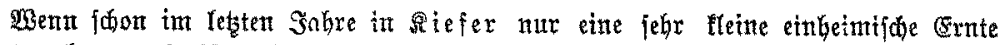

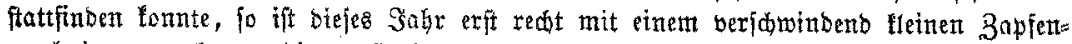

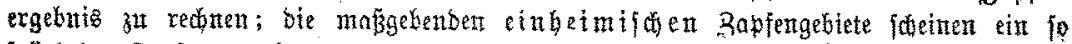

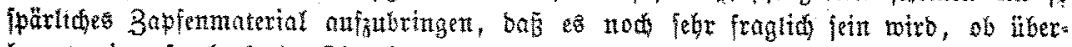
baupt eine fortlaufenbe Rlenglompagnte nit bentiden Bapfen ftattfinben font, unt

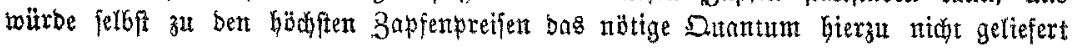

